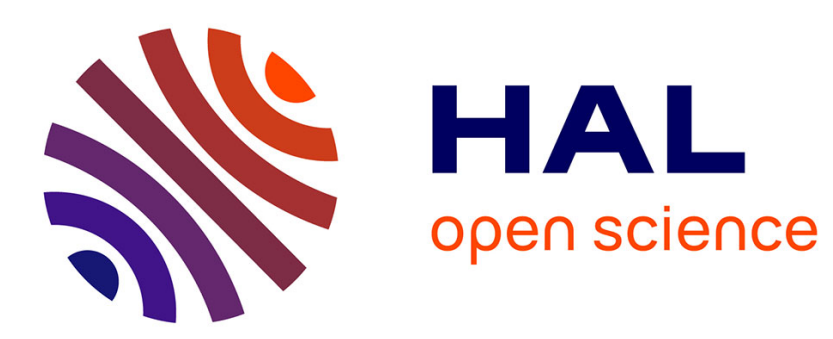

\title{
Heterogeneous disconnection nucleation mechanisms during grain boundary migration
}

\author{
Nicolas Combe, Frédéric Mompiou, M Legros
}

\section{To cite this version:}

Nicolas Combe, Frédéric Mompiou, M Legros. Heterogeneous disconnection nucleation mechanisms during grain boundary migration. Physical Review Materials, 2019, 3 (6), 10.1103/PhysRevMaterials.3.060601 . hal-02117724

\section{HAL Id: hal-02117724 \\ https://hal.science/hal-02117724}

Submitted on 2 May 2019

HAL is a multi-disciplinary open access archive for the deposit and dissemination of scientific research documents, whether they are published or not. The documents may come from teaching and research institutions in France or abroad, or from public or private research centers.
L'archive ouverte pluridisciplinaire HAL, est destinée au dépôt et à la diffusion de documents scientifiques de niveau recherche, publiés ou non, émanant des établissements d'enseignement et de recherche français ou étrangers, des laboratoires publics ou privés. 


\title{
Heterogeneous disconnection nucleation mechanisms during grain boundary migration
}

\author{
N. Combe, $, 1,2$, F F Mompiou, ${ }^{1,2}$ and M. Legros ${ }^{1,2}$ \\ ${ }^{1}$ Centre d'Elaboration de Matériaux et d'Etudes Structurales, CNRS UPR 8011, \\ 29 rue J. Marvig, BP 94347, 31055 Toulouse cedex 4, France \\ ${ }^{2}$ Université de Toulouse ; UPS ; F-31055 Toulouse, France
}

(Dated: March 18, 2019)

\begin{abstract}
Shear-coupled grain boundary (GB) migration has been evidenced as an efficient mechanism of plasticity in absence of dislocation activity. The GB migration occurs through the nucleation and motion of disconnections. Using molecular simulations, we report a detailed study of the elementary mechanisms occurring during heterogeneous disconnection nucleation. We study the effect of a preexisting sessile disconnection in a symmetric $\Sigma 17(410)$ [001] tilt GB on the GB migration mechanism. Shearing this imperfect GB induces its migration and reveals a new GB migration mechanism through the nucleation of a mobile disconnection from the sessile one. Energy barriers and yield stress for the GB migrations are evaluated and compared to the migration of a perfect GB. We show that the migration of the imperfect GB is easier than the perfect one and that a sessile disconnection can operate as a source of disconnection driving the GB migration. This GB migration mechanism has been observed on two other high-angle GB.
\end{abstract}

The plastic deformation of polycrystalline solids is generally due to the mobility of dislocations. Grain boundaries (GB) are considered as static obstacles to mobile dislocations as drawn by the Hall-Petch law [1]. Consequently, in nano-crystalline materials (grain sizes $<100 \mathrm{~nm}$ ), the dislocation-mediated plasticity is reduced or even absent. Recent results have evidenced that in such cases, GB under stress can migrate and participate to the plastic deformation [2 [5] .

Among the possible GB-based mechanisms [6], both experiments [2, 7-10] and molecular dynamics simulations [11 13] have evidenced the shear-coupled GB migration (SCGBM) as an efficient plastic mechanism at low temperature for low- and high-angle GB. The application of a shear stress on the GB induces its migration (over a distance $m$ ) coincidently with the relative in-plane translation $d$ of the two grains forming the GB. The coupling factor $\beta=\frac{d}{m}$ characterizes the migration.

The SCGBM has been abundantly studied using atomistic simulations investigating for instance the dependence of the GB mobilities on the GB misorientation or structure 11, 13 15. The elementary mechanisms of SCGBM have been numerically investigated on flat perfect model GB: the GB migration results from the nucleation of two mobile disconnections with opposite Burgers vectors (BV) and their migration in opposite directions 16 23]. Disconnections are GB line defects that have both step (height) and dislocation character 24]. Various experiments have given strong clues of the role of these disconnections in the SCGBM [9, 10].

However, real GB are far from being flat and perfect, and the role of pre-existing defects as GB steps or triple junctions [25, 26] has been considered on the GB migration. In the presence of defects, the nucleation of disconnections will certainly be heterogeneous. In this study, we investigate the heterogeneous disconnection nucleation in presence of a pre-existing disconnection with a BV out of the GB plane. Indeed, bulk dislocations interact with $\mathrm{GB}$ through various processes [27, 28]: they can be transmitted or absorbed, totally or partially. In case of a total or partial absorption, a residual disconnection remains in the GB. While glissile disconnections quickly move as they easily couple to a shear stress, disconnections with a BV out of the GB plane are sessile and potentially affect the GB migration. We examine here this latter case and reveal a new SCGBM mechanism through the nucleation of mobile disconnections from a sessile one.

As a representative and simple case, we study the migration of a symmetric tilt GB in response to an external shear deformation in a copper bi-crystal using the molecular dynamics (MD) simulation package LAMMPS 29] and an embedded-atom potential 30]. Figure 1a reports a sketch of the simulation cell under study. Initially, the simulation cell is prepared with two symmetric grains of a perfect fcc copper crystal disorientated relatively to each other by an angle $\theta=28.07^{\circ}$ around the [001] direction and separated by a perfect $\Sigma 17(410)[001]$ GB. Periodic boundary conditions (PBC) are applied in the [140] (y-axis) and [001] (z-axis) directions. The simulation cell $\mathrm{x}$ ( $\mathrm{x}$ direction along [410]), $\mathrm{y}$ and $\mathrm{z}$ sizes are, respectively, $32.4 \mathrm{~nm}, 11.9 \mathrm{~nm}$, and $2.9 \mathrm{~nm}$. The cell contains 94216 atoms. Two $1.5 \mathrm{~nm}$ thick slabs at the top and bottom of the cell that contain atoms with relative positions frozen to the perfect lattice ones are used to impose a shear stress on the GB. Equilibrium structures of the system are obtained by minimization of the potential energy at $0 \mathrm{~K}$ using a conjugated gradient algorithm. In order to create the pre-existing disconnection with a realistic structure, a $1 / 2[110]$ edge bulk dislocation is artificially introduced in grain 1 (Fig. 17). This dislocation is moved towards the GB by the application of a stress and is fully absorbed in the GB: it creates a disconnection, referred as $\delta$ in the following. 31] Fig. [1-i) reports 
the atomic configuration of the disconnection $\delta$. The BV of the disconnection $\delta$ is the same as the $1 / 2[110]$ bulk dislocation 32 and has both normal and coplanar components to the GB plane. Fig.1] reports this BV in the dichromatic pattern. Due to the normal component of the $\mathrm{BV}$, this disconnection $\delta$ is sessile, as seen in any of our simulations.

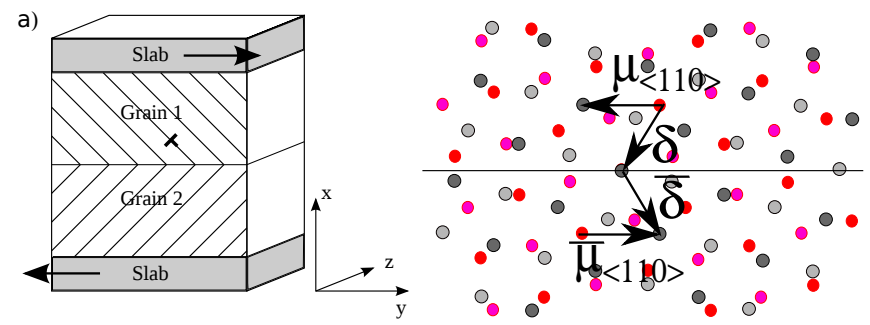

C) i)
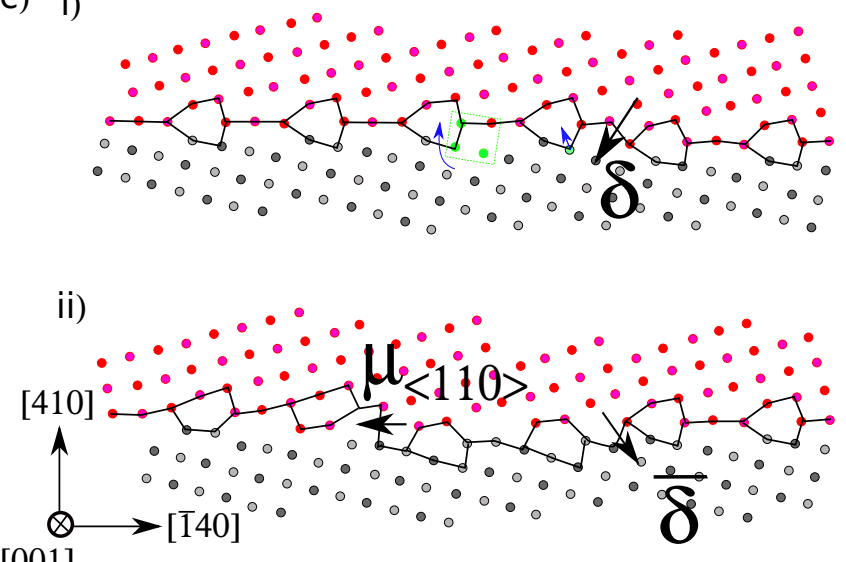

[001]

FIG. 1. (color online) a) Sketch of the simulation cell before the absorption of the dislocation used to create a pre-existing disconnection. b) Dichromatic pattern of $\Sigma 17(410)[001]$ GB evidencing the BV of the disconnections $\delta, \bar{\delta}, \mu_{\langle 110\rangle}$ and $\bar{\mu}_{\langle 110\rangle}$. The horizontal solid line evidences the GB interface. c) i) Configuration of the system $(\mathrm{GB}+$ disconnection $\delta)$. Green atoms and blue arrow indicate the main atomic displacement inducing the nucleation of $\bar{\delta}$ and $\mu_{\langle 110\rangle}$ disconnections. ii ) Configuration for $\mathrm{RC}=0.22$ and $\mathrm{d}=0.57 \mathrm{~nm}$ along the MEP after the decomposition of the disconnection $\delta$ into $\bar{\delta}$ and $\mu_{\langle 110\rangle}$. The black solid lines, a guide to the eyes reveal the structural units. Black (Grey) and Red (Pink) atoms belong to different grains. Black(Red) and Grey(Pink) atoms have not the same z-coordinate.

The SCGBM of this system $(\mathrm{GB}+$ disconnection $\delta)$ is studied at $0 \mathrm{~K}$ : the slabs are translated relatively to each other in the y-direction by small increments $(0.002 \mathrm{~nm})$. At each step, the potential energy is minimized. Fig. 2a reports the shear stress (calculated using the virial stress tensor) as a function of the relative shear displacement $d$ of the slab (for both positive and negative displacements). The shear stress is a linear function of $d$ in the elastic regime. Shear stress discontinuities correspond to plastic events namely GB migrations here. For the system $(\mathrm{GB}+$ disconnection $\delta)$, the GB migrates at $d=-0.82 \mathrm{~nm}$ in the $[410](\mathrm{x})$ direction for a yield stress $-1.68 \mathrm{GPa}$ and at $d=0.65 \mathrm{~nm}$ in the $[\overline{4} \overline{1} 0](-\mathrm{x})$ direction for a yield stress 1.3 GPa.
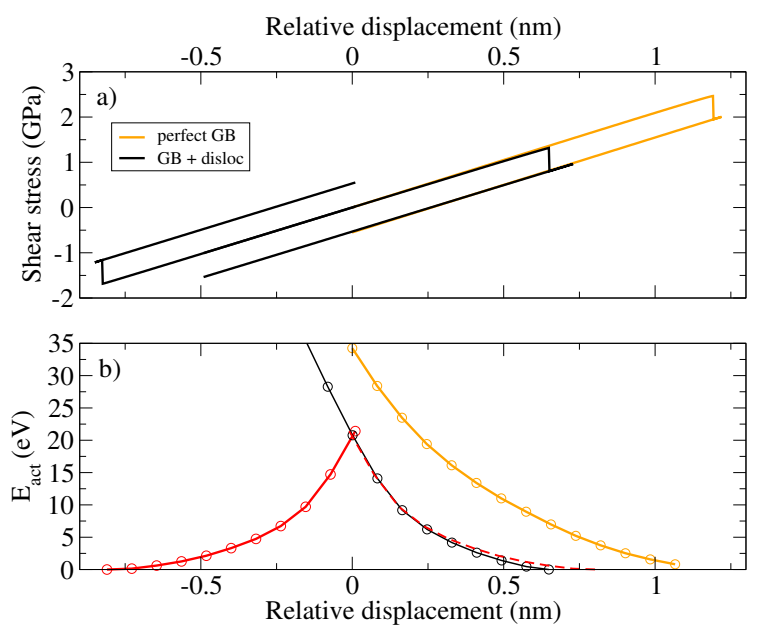

FIG. 2. a) Shear stress and b) activation energies as a function of the relative shear displacement at $0 \mathrm{~K}$ for the system (GB+disconnection $\delta$ ) (black solid line) and for a perfect $\Sigma 17(410)[001]$ GB (orange curve). b) The black (red) solid line corresponds to the migration of the system $(\mathrm{GB}+\operatorname{disconnection} \delta)$ in $[\overline{4} \overline{1} 0]([410])$ direction. The dashed red line corresponds to the symmetric of the red solid line compared to $d=0$ and corresponds the activation energies of the SCGBM of a system $(\mathrm{GB}+$ disconnection $\bar{\delta})$ for positive shear.

For comparison, Fig. 2a) also reports the same curve for a perfect $\Sigma 17(410)[001]$ GB (for positive displacement only since this curve is symmetric): the measured yield stress of the perfect $\Sigma 17(410)[001] \mathrm{GB}$ is $2.45 \mathrm{GPa}$ and occurs at $1.22 \mathrm{~nm}$. The decrease of the yield stress in presence of a disconnection $(\delta)$ evidences the role of the sessile disconnection on the SCGBM mechanism, and thus the relevance of our study [17]. The $\delta \mathrm{BV}$ is asymmetric compared to a positive or a negative shear, explaining the difference in yield stresses for positive and negative $d$ : both migrations (at $d=-0.82 \mathrm{~nm}$ and $d=0.65 \mathrm{~nm}$ ) do not occur following the same mechanism as revealed below. After the GB migration, the GB configuration is similar to its one before migration, it still contains the disconnection $\delta$ at the same position along the $\mathrm{y}$-direction.

In order to reveal the SCGBM mechanisms, the system after the GB migration is unloaded (Fig. 2a) to generate configurations of the system after the GB migration for various values of $d$. Nudge Elastic Band (NEB) calculations [33] are performed between configurations before and after the GB migration at given $d$ as a function of a reaction coordinate $(\mathrm{RC})$ 34. NEB calculations have been performed with 60 images, except few NEB calculations that have been performed with 160 images to check the reliability of the results compared to the number of NEB images. Below, we first address the mecha- 
nism involved by the GB migration in the $[\overline{4} \overline{1} 0](-\mathrm{x})$ direction investigating the minimum energy path (MEP) between the initial and final configurations. The MEP (not shown) is smooth and does not show any metastable state.

Fig. 1-ii) reports an atomic configuration along the MEP $(\mathrm{d}=0.57 \mathrm{~nm})$ at $\mathrm{RC}=0.22$ : the SCGBM occurs through the decomposition of disconnection $\delta$ into two disconnections referred as $\bar{\delta}$ and $\mu_{\langle 110\rangle}$ 35]. Disconnections $\bar{\delta}$ and $\mu_{\langle 110\rangle}$ are respectively sessile and mobile. Disconnection $\mu_{\langle 110\rangle}$ travels through the simulation cell (in the [1 $1 \overline{4} 0](-y)$ direction) and finally recombines (due to $\mathrm{PBC}$ ) with $\bar{\delta}$ to recreate the disconnection $\delta$. Fig. [3b) sketches this mechanism of GB migration through heterogeneous nucleation of disconnections. The BV of disconnections $\delta, \bar{\delta}$, and $\mu_{\langle 110\rangle}$ are reported in the dichromatic pattern of Fig 1 b. Both conservations of BV and step heights are ensured: the vectorial and algebraic sums of the BV and heights of $\bar{\delta}$ and $\mu_{\langle 110\rangle}$ correspond to the ones of $\delta$. The height of disconnection $\delta$ is half the one of $\mu_{\langle 110\rangle}$ 24] and is the opposite of the one of $\bar{\delta}$. The $\bar{\delta} \mathrm{BV}$ has a normal component relative to the GB plane and $\bar{\delta}$ is thus sessile. The disconnection $\mu_{\langle 110\rangle}$ corresponds to the disconnection nucleated during the $\langle 110\rangle$ GB migration mode. The absence of metastable state along the MEP agrees with the previous calculations on a perfect GB for the $\langle 110\rangle$ mode [20].

Examining the configuration along the MEP, a shuffling mechanism revealed in Fig. 1 $\mathrm{b}-\mathrm{i}$ ) is responsible for the nucleation of the disconnections $\bar{\delta}$ and $\mu_{\langle 110\rangle}$ from $\delta$ : the main atomic displacements responsible for this nucleation are mentioned in Fig. 15-i). The motion of the $\mu_{\langle 110\rangle}$ disconnection is also produced by a shuffling mechanism already reported in the literature [20].

In order to complement our characterization of the GB migration through heterogeneous nucleation of disconnections, Fig. 20) reports the activation energies calculated from the MEP for different values of the shear displacement $d$. Activation energies are here defined as the difference between the maximum energy along the MEP and the energy of the initial equilibrium configuration (before $\mathrm{GB}$ migration i.e. $\mathrm{RC}=0$ ). These activation energies have been calculated for both the $(\mathrm{GB}+$ disconnection $\delta)$ system (black curve) and for the perfect GB (orange curve). For the perfect GB, the GB migration occurs through the homogeneous nucleation and motions of two mobile disconnections $\mu_{\langle 110\rangle}$ and $\bar{\mu}_{\langle 110\rangle}$ following the mechanism sketched in Fig [3a) [18]. The BV and step height of the $\bar{\mu}_{\langle 110\rangle}$ disconnection are opposite to the one of $\mu_{\langle 110\rangle}$ (see Fig. 1b). As expected, activation energies decrease with increasing shear displacement and cancel for shear displacements corresponding to the spontaneous GB migration at $0 \mathrm{~K}$ (at the yield stress), respectively at $d=0.65 \mathrm{~nm}$ and $d=1.22$ $\mathrm{nm}$ for the $(\mathrm{GB}+$ disconnection $\delta)$ system and for the perfect GB. At given shear displacement, the activation en-

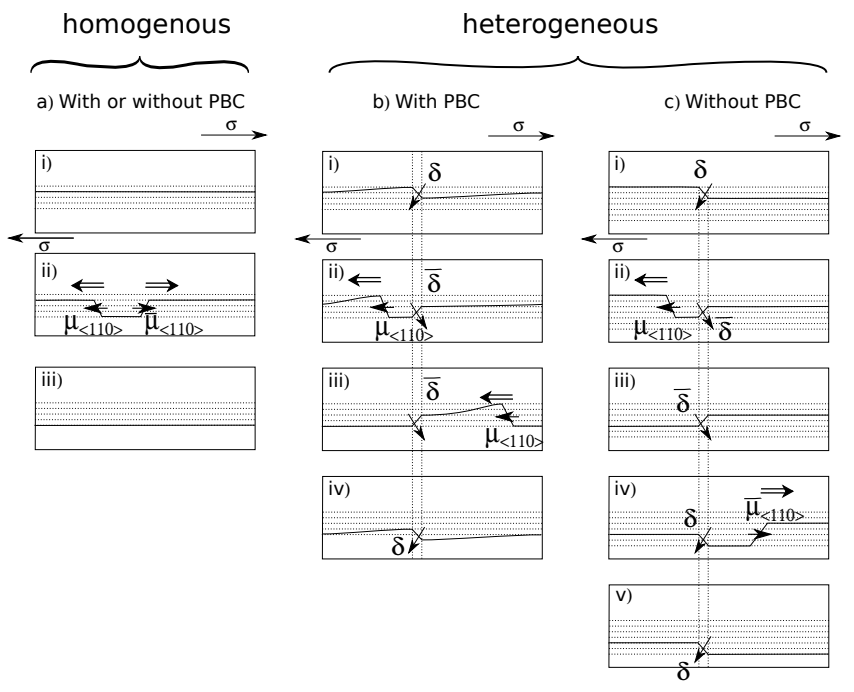

FIG. 3. (color online) a) Mechanism of homogeneous disconnections nucleation. b) and c) Mechanism of heterogeneous disconnections nucleation with and without PBC. The solid lines feature the GB interface. Dashed lines are equidistant in order to reveal the disconnection heights. Double and solid arrows indicate the motion and the BV of disconnections.

ergy for the perfect GB migration is clearly much higher than the one for the $(\mathrm{GB}+$ disconnection $\delta$ ) system (for instance, for $d=0.25 \mathrm{~nm}$, it is more than 3 times higher). The migration of a GB is easier in the presence of a preexisting sessile disconnection [17, 35].

The mechanism of Fig. Bb) is related to the presence of PBC. Indeed, PBC implicitly involve a network of disconnections $\delta$. Therefore, the nucleated disconnection $\mu_{\langle 110\rangle}$ moves away from the disconnection $\bar{\delta}$ and recombine with a PBC image of $\bar{\delta}$ as sketched in Fig. Bb).

In order to address the case of a realistic sample, we have performed a large MD simulation at $0 \mathrm{~K}$ in a cell without PBC involving about 350000 atoms. We have observed the following full SCGBM mechanism for heterogeneous nucleation of disconnection [35]. The disconnection $\delta$ first decomposes in a mobile $\mu_{\langle 110\rangle}$ and a sessile $\bar{\delta}$ disconnection, the disconnection $\mu_{\langle 110\rangle}$ migrates away in the $[1 \overline{4} 0](-y)$ direction. The $\bar{\delta}$ disconnection then decomposes into a mobile $\bar{\mu}_{\langle 110\rangle}$ that migrates away in the [140] (y) direction and a $\delta$ disconnection . The (GB+ disconnection $\delta$ ) system is hence restored after its migration. Fig. 3r) reports this full SCGBM mechanism. Since both mobile disconnections $\mu_{\langle 110\rangle}$ and $\bar{\mu}_{\langle 110\rangle}$ are characteristic of the $\langle 110\rangle$ mode, the apparent coupling factor is the $\langle 110\rangle$ mode one: $\beta=6 / 5$.

We have energetically characterized the decomposition of the disconnection $\bar{\delta}$. Since the effect of a (positive) shear stress on disconnection $\bar{\delta}$ is equivalent to impose a negative shear stress on disconnection $\delta$, we have investigated the mechanism involved by the migration of the system $(\mathrm{GB}+$ disconnection $\delta)$ at $0 \mathrm{~K}$ at $d=-0.82 \mathrm{~nm}$ in Fig. 22). Performing NEB calculations between configu- 
rations before and after GB migration at $d=-0.82 \mathrm{~nm}$, we have retrieved the expected mechanism described above. Fig. 2b) reports the activation energies (red solid lines) extracted from these NEB calculations for the SCGBM of the system $(\mathrm{GB}+$ disconnection $\delta)$ for negative values of $d$. The symmetric of this curve compared to $d=0$ (red dashed line) corresponds to the activation energies for the SCGBM of the system (GB+disconnection $\bar{\delta})$ for positive values of $d$. From Fig. 2 $b$ ), the activation energies for both systems $(\mathrm{GB}+$ disconnection $\delta)$ and $(\mathrm{GB}+$ disconnection $\bar{\delta})$ are comparable and only significantly differs for $d>0.5 \mathrm{~nm}$.

The $\Sigma 17(410)$ [001] GB has a misorientation angle of $\theta=28.07^{\circ}$ around the [001] direction. In order to check the robustness of the proposed full SCGBM mechanism for heterogeneous nucleation in the presence of a $\delta$ disconnection, we have performed MD simulations without PBC for two high-angle GB $\Sigma 25(430)[001]\left(\theta=73.74^{\circ}\right)$ and $\Sigma 41(540)[001]\left(\theta=77.32^{\circ}\right)$. At both $0 \mathrm{~K}$ and $300 \mathrm{~K}$, we observe the nucleation of disconnections $\mu_{\langle 110\rangle}$ and $\bar{\mu}_{\langle 110\rangle}$ from the disconnection $\delta$ and their propagation in opposite direction. While at $0 \mathrm{~K}$, we observe the simultaneous nucleation and motion of the two disconnections [36], at $300 \mathrm{~K}$, the ones of the disconnections $\mu_{\langle 110\rangle}$ clearly first occur before the ones of $\bar{\mu}_{\langle 110\rangle}$ in full agreement with the mechanism reported in Fig. [35).

As a conclusion, a $\Sigma 17(410)[001]$ GB with a preexisting disconnection $\delta$ can shear-couple and migrate. Our work evidences that sessile disconnections can act as sources of mobile disconnections $\mu_{\langle 110\rangle}$ and $\bar{\mu}_{\langle 110\rangle}$. The presence of a disconnection source significantly decreases the yield stress as well as the activation energy barrier for the SCGBM. This GB migration mechanism reported in Fig. 35) is robust as it has been observed in two other high-angle GB at both null and finite temperatures. Several perspectives to this work can be considered. Other disconnections different from the one studied in this work could be considered: especially, from the dichromatic pattern and the decomposition mechanism, the $\delta$ disconnection is related to the $\langle 110\rangle$ mode. The study of the SCGBM of GB including disconnections favoring other modes would be highly relevant in order to investigate if the presence of a sessile disconnection could favor a specific mode. Besides, the shearing of a low-angle GB $\Sigma 37(610)[001]$ incorporating a $\delta$ disconnection without $\mathrm{PBC}$ at both $0 \mathrm{~K}$ and $300 \mathrm{~K}$ has not shown the mechanism Fig. 3r) cited above but a more complex one presumably related to the preferential activation of the $\langle 100\rangle$ mode for low-angle perfect GB at finite temperature [11]. An investigation of the competition between temperature effects that favor $\langle 100\rangle$ mode and the presence of a $\delta$ disconnection that favor the $\langle 110\rangle$ mode would be highly relevant for low-angle GB.

This work was performed using HPC resources from CALMIP (Grant No. 2012-12172) and was funded by ANR-17-CE08-0007.
* nicolas.combe@cemes.fr

[1] E. Hall, Proc. Phys. Soc. B 64, 747 (1951).

[2] M. Legros, D. Gianola, and K. Hemker, Acta Mat. 56, 3380 (2008)

[3] M. Jin, et al. Acta Mat. 52, 5381 (2004).

[4] T. J. Rupert, et al. Science 326, 1686 (2009).

[5] J. Schäfer and K. Albe, Acta Mat. 60, 6076 (2012)

[6] M. A. Meyers, A. Mishra, and D. J. Benson, Prog. Mat. Sci. 51, 427 (2006).

[7] K. Zhang, J. R. Weertman, and J. A. Eastman, Appl. Phys. Lett. 87, 061921 (2005).

[8] T. Gorkaya, D. A. Molodov, and G. Gottstein, Acta mat. 57, 5396 (2009).

[9] A. Rajabzadeh, et al., Phil. Mag. 93, 1299 (2013)

[10] Q. Zhu, et al., Nature Comm. 10, 156 (2019).

[11] J. W. Cahn, Y. Mishin, and A. Suzuki, Acta Mat. 54, 4953 (2006).

[12] M. Velasco, H. V. Swygenhoven, and C. Brandl, Script. Mat. 65, 151 (2011).

[13] E. R. Homer, et al., Acta Mat. 61, 1048 (2013)

[14] Y. Mishin, et al., Phys. Rev. B 75, 224101 (2007)

[15] T. Frolov, et al., Nature Com. 4, 1899 (2013).

[16] L. Wan and S. Wang, Phys. Rev. B 82, 214112 (2010).

[17] H. Khater, et al., Acta Mat. 60, 2007 (2012)

[18] A. Rajabzadeh, et al., Phys. Rev. Lett. 110, 265507 (2013)

[19] C. P. Race, J. von Pezold, and J. Neugebauer, Phys. Rev. B 89, 214110 (2014).

[20] N. Combe, F. Mompiou, and M. Legros, Phys. Rev. B 93, 024109 (2016)

[21] O. MacKain, et al., Phys. Rev. B 95, 134102 (2017)

[22] N. Combe, F. Mompiou, and M. Legros, Phys. Rev. Mat. 1, 033605 (2017).

[23] J. Han, S. L. Thomas, and D. J. Srolovitz, Progress in Materials Science 98, 386 (2018)

[24] J. Hirth and R. Pond, Acta Mat. 44, 4749 (1996).

[25] M. Aramfard and C. Deng, Modelling Simul. Mater. Sci. Eng. 22, 055012 (2014)

[26] R. Hadian, et al., Phys. Rev. Mat. 2, 043601 (2018).

[27] M. Chassagne, M. Legros, and D. Rodney, Acta Mat. 59, 1456 (2011)

[28] M. Dupraz, et al., Acta Materialia 144, 68 (2018).

[29] S. J. Plimpton, J. Comp. Phys. 117, 1 (1995)

[30] Y. Mishin, et al., Phys. Rev. B 63, 224106 (2001)

[31] Our procedure is pseudo-realistic: indeed, a 1/2[110] edge bulk dislocation normally decomposes in partial Shockley ones: in our case, because simulations are performed at 0 $\mathrm{K}$, this decomposition was not observed. However, even in the case of a decomposition, a relevant stress would drive the two partial dislocations in the GB yielding the creation of the $\delta$ disconnection.

[32] Elastic distortions are necessary in order to satisfy the $\mathrm{PBC}$ in the $\mathrm{y}$-direction: the GB interface is slightly curved away from the disconnection vicinity.

[33] G. Henkelman, B. P. Uberuaga, and H. Jonsson, J. Chem. Phys. 113, 9901 (2000)

[34] The RC is here defined as the cumulative distance (normalized by the total cumulative distance) between adjacent replicas in the configuration space of dimension $3 \mathrm{~N}$ with $\mathrm{N}$ the number of atoms.

[35] A. Serra and D. J. Bacon, 
Philosophical Magazine A 73, 333 (1996).

[36] Despite our efforts, we have not been able to evidence their successive nucleations and propagations. 